\title{
El MÁS ACÁ DE LA MODERNIDAD LOS USOS DE LA MODERNIDAD COMO TEMPORALIDAD RETÓRICA
}

\author{
Steffan Igor Ayora Diaz \\ Gabriela Vargas Cetina
}

\author{
Volar al Este era desplazarse velozmente \\ hacia el futuro — las horas impulsadas por \\ el Jet corrian apresuradamente, el día \\ siguiente llegó sobre las alas- \\ pero se sentía como un regreso al pasado. ...
}

$\mathrm{D}$ e esta manera Rushdie (2001:236) describe las vivencias del profesor Malik Solanka mientras viaja al pequeño país Lilliput-Blefescu, donde los revolucionarios han tomado el poder portando máscaras que reproducen y permiten actuar el simulacro estructurado por un juego virtual localizado en la Red Amplia Global (el www). El grupo revolucionario en el poder ha desplazado a otro grupo que mantenía a las clases desposeídas en un régimen feudal y, para mantenerse en el poder, toma medidas también totalitarias. Un pequeño país que nunca fue moderno se encuentra en la vorágine de la posmodernidad: cadenas de significantes separadas del significado original contribuyen a construir el sentido de la nueva coyuntura social. La cita, aun cuando extraída de un trabajo de ficción, nos invita a adelantar algunas preguntas: ¿dónde y en qué tiempo se encuentran la premodernidad y la posmodernidad? Si el personaje vuela hacia el pasado, ¿cómo puede encontrar en éste el futuro, la posmodernidad?

En este ensayo proponemos que es necesario repensar, de manera crítica, los modelos que actualmente utilizamos para pensar en modernidad, posmodernidad

Steffan Igor Ayora Diaz y Gabriela Vargas Cetina, Facultad de Ciencias Antropológicas, Universidad Autónoma de Yucatán. y tradición para poder escapar a las trampas conceptuales implícitas en estos conceptos, cargados de un fuerte contenido historicista. Este es el espíritu que anima nuestra presente reflexión. Queremos argumentar aquí que la familia de conceptos 'posmodernidad', 'posmodernismo' y 'posmodernización' tiene sentido como equivalente, en cierta medida, a otras familias en las que el eje puede ser constituido sea por modernidad, modernidad reflexiva, modernidad tardía, alta modernidad, hipermodernidad o supermodernidad. Estos grupos de conceptos deben ser vistos como herramientas conceptuales útiles en los esfuerzos por comprender (en su sentido hermenéutico) la vida en la sociedad contemporánea; es decir, tanto las características ('objetivas') socioestructurales y culturales que constituyen el ambiente en el que los individuos se desplazan e interactúan, como la experiencia (inter/subjetiva y estética) de los individuos que habitan el mundo.

Reconociendo que los conceptos son los anteojos conceptuales que median nuestra percepción y comprensión del mundo social, nuestra propuesta es que para poder utilizar estos conceptos de manera que contribuyan a nuestro análisis de la sociedad y culturas contemporáneas es necesario despojarlos de sus connotaciones 
temporales y espaciales resaltando, precisamente, las implicaciones políticas (en el sentido que se deriva del neocolonialismo cultural) que esos conceptos tienen. Aunque en otros momentos hemos encontrado necesario hacer referencia a una cierta condición cultural posmoderna, en el presente artículo sostenemos que la noción de modernidades locales puede ser de gran utilidad para describir y explicar la naturaleza de distintos fenómenos contemporáneos. Sin embargo, nosotros consideramos que es necesario privar al concepto de modernidad de su sentido alocrónico (Fabian 1983); es decir, del poder retórico que el concepto tiene para desplazar y colocar a diferentes sociedades y culturas, en forma simultánea, a unas más en el pasado y a otras más en el futuro. Por medio de esta redimensionalización, la idea de la modernidad como algo que podemos 'individuar' teóricamente se disuelve, se fragmenta y pierde su sentido normativo. Por tanto, proponemos que una reflexión crítica acerca de los significados que se derivan de nuestro lenguaje disciplinario debe de llevarnos a modificar nuestras estrategias interpretativas y, finalmente, textual-descriptivas en el momento de producir nuestras etno-grafías.

Es común pensar a la modernidad como un proceso caracterizado por un eje temporal y uno espacial. La modernidad se alcanza únicamente, según una cierta concepción reduccionista, al trascender la cosmovisión cultural y las estructuras sociales estáticas de la tradición. Una sociedad tradicional, entonces, estaría orientada hacia el pasado, o ligada a él; mientras que una sociedad moderna estaría orientada hacia el futuro y definida por una orientación hacia el cambio permanente que se traduce en que lo sólido se desvanezca en el aire. Esta comprensión tiene como una de sus consecuencias la negación (discursiva y práctica) de la coexistencia temporal (coevalidad, según Fabian 1983) de grupos sociales que habitan el mundo al mismo tiempo.

Según otro malentendido, la modernidad estaría ligada de manera inextricable a la sociedad occidental.
Desde este punto de vista, por una parte, es en el mundo occidental, europeo y norteamericano, donde se gesta y desarrolla la modernidad y de ahí ésta se desplaza hacia nuevos territorios. Por otra parte, y con base en la premisa anterior, se sostiene la ilusión de que, para que una sociedad se modernice, deberá occidentalizarse; es decir, mientras más se parezca una sociedad no occidental a aquéllas occidentales, más moderna será. Una sociedad distinta de la occidental sería, en consecuencia, tradicional. Said (1978) ha discutido ya las consecuencias retóricas y políticas (por ejemplo, la estructura de dominación y subordinación colonial cultural) que el orientalismo tiene en las relaciones entre distintas sociedades (para Latinoamérica, Moreiras 1998, usa el cognado 'latinoamericanismo').

Es claro que la distinción entre una sociedad moderna y una tradicional tiene funciones alterizantes que sostienen y legitiman distintas formas de colonialismo y neocolonialismo (cultural, político, económico). Sería entonces importante reconocer que existe un orden globalizado cuya estructura de fuerzas es desigual y que beneficia y subordina a distintas sociedades. Lo tradicional solamente puede ser pensado en relación con lo moderno; es decir, cada uno es producto del otro y cada uno necesita del otro para autocomprenderse. Es porque existen sociedades urbanas, opulentas, consumistas, usuarias de (rápidamente cambiantes) tecnologías muy costosas, y racionalizadas (en resumen, sociedades generalmente vistas como modernas), que existen grupos sociales rurales empobrecidos que deben producir en condiciones tecnológicas y de conocimiento distintas y desventajosas en relación con las primeras (es decir, sociedades que son vistas como tradicionales). Necesitamos reconocer que las condiciones históricas y estructural-funcionales que propician ambas formaciones socioculturales son las mismas. La tradición nace junto con, y está ligada a, la modernidad y lo que hoy muchos identifican como posmodernidad. En consecuencia, la discusión de si la modernidad comien- 
za en tal o cual fecha, si la tradición termina o no ese mismo día, y si la posmodernidad puede existir en una sociedad que nunca fue moderna, nos conduce únicamente a callejones sin salida, poco productivos conceptualmente, aunque favorezcan la impresión de miles de páginas en publicaciones académicas.

Más aún, usado de esta forma, el concepto de modernidad tiene implicaciones políticas que subordinan retóricamente unas formas de saber a otras y que justifican la colonización material, intelectual y epistemológica de unas sociedades por otras. La categoría de modernidad, con sus implicaciones de tiempo futuro y de un tipo de racionalidad superior a todo lo que no sea moderno, transforma todos los saberes 'premodernos' en 'atrasados'e 'irracionales'. El resultado concreto de estos procesos discursivos es la implementación de estrategias de 'modernización' que aparecen como universalmente deseables para todas las sociedades. Una de las herramientas discursivas más poderosas dentro del complejo retórico apuntalado por la idea de modernidad es el concepto de 'desarrollo'. El desarrollo es visto como la vía de ecualización de los tiempos aparentemente diferentes en los que viven las distintas sociedades: por medio del desarrollo, que en términos prácticos se traduce en 'planes y proyectos de desarrollo', unas sociedades pueden salir de su 'atraso' y 'alcanzar' las dimensiones tecnológicas y temporales en las que otras, supuestamente 'más avanzadas' (es decir, 'más modernas') ya se desenvuelven. Antropólogos y antropólogas que se preocupan por el estudio de los proyectos de desarrollo socioeconómico han apuntado ya los efectos retóricos y las consecuencias materiales que la persecución de la modernización por medio del desarrollo traen consigo: la despolitización a nivel retórico de las decisiones políticas, la destrucción de las lógicas económicas no basadas en relaciones monetarias, la desposesión de grupos enteros de sus saberes locales y de sus recursos - que pasan a ser administrados por el Estado o por agencias internacionales que 'saben' mejor que la gente local cómo deben de ser usados y administrados — y, en muchos casos, la destrucción de modos de vida que, aunque poco rentables, habían permitido a muchas poblaciones humanas subsistir quizá por siglos en ambientes extremos (Escobar 1995, Fairhead 2000, Ferguson 1994, Hobart, ed. 1993, Latham 2000, Leys 1996). Por tanto, el concepto de modernidad, en su acepción cronotópica, no puede seguir siendo visto como inocente, sino como eminentemente político.

Si prescindimos de las dimensiones espaciales y temporales, ¿qué utilidad se puede encontrar en los conceptos sea de 'tradición', 'modernidad' o 'posmodemidad'? En concreto, nuestra postura parte de que es necesario eliminar esta distinción que instituye, legitima y distribuye desigualmente el poder entre las sociedades que jerarquiza, para así poder eliminar del discurso antropológico la dimensión alocronizante que esta jerarquía implica. Esta necesidad debe encontrar su balance con la experiencia subjetiva e intersubjetiva de las condiciones de vida cotidianas que los sujetos definen, como resultado de su experiencia, sea como tradicionales, modernas o posmodernas. Después de todo, el discurso ideológico gestado entre, y propagado por, las elites académicas y políticas, ha contribuido a hacer de estas categorías elementos básicos para entender la propia vida en sociedad. Es aquí donde el papel de la antropología, como disciplina de lo cotidiano, se vuelve fundamental.

Nuestra propuesta es que necesitamos comenzar a pensaren modernidades locales. Como argumentamos más adelante, este concepto permite tomar en cuenta las dinámicas local-globales y, al mismo tiempo, nos ayuda a entender las formas intersubjetivas en las que los individuos construyen el sentido de sus propias vidas y de la sociedad que habitan. Más que una revolución radical en la forma de hacer trabajo de campo antropológico, se trataría, entonces, de reconceptualizar y reinterpretar los elementos sociales, económicos, culturales y políticos que entran en juego, de diversas maneras, en estas múltiples articulaciones de lo global y lo local. Así, existe 
aún la posibilidad (cuando los financiamientos lo permiten) de mantener el trabajo de campo a profundidad en una sola comunidad, por un largo tiempo (como proponen, por ejemplo, Englund y Leach 2000), o enfocarse sobre ese aspecto que ha sido 'objeto' tradicional de la antropología, el ritual, como proponen Jean y John Comaroff (1993) pensando en él, no como sitio privilegiado de la tradición, sino como ese espacio creativo en donde lo global y lo local se funden. Más aún, es quizá necesario seguir pensando en las consecuencias de la translocalidad que generan los procesos de globalización y fomentar estudios multisituados, tal como ya ha propuesto Marcus (1998). De esta manera, sin transformar necesariamente los métodos generales de hacer el trabajo de campo, lo que necesitamos es repensar y redimensionar los 'datos' que ahí (o aquî) encontramos en términos de la multiplicidad de posibles formas que lo moderno puede tomar en distintas localidades (ver también Knauft, ed. 2002).

En este sentido, al mismo tiempo que el concepto de 'modernidades locales' nos ayuda a abolir analíticamente la distinción entre sociedades tradicionales, modernas y posmodernas, nos permite avanzar en el camino hacia unas ciencias sociales que reconozcan el sentido cotidiano que los sujetos le dan a esos términos en su vida diaria y evitar las trampas de los orientalismos y los alocronismos fáciles. En vez de buscar las raíces de la diferencia entre formas sociales y culturales en la localización espaciotemporal de los grupos humanos, necesitaremos explicar cómo estructural e históricamente se han gestado distintas modernidades locales.

En la siguiente sección de este ensayo discutimos brevemente una serie de conceptos que han sido inscritos tanto en descripciones de la condición posmoderna como de distintas modernidades adjetivadas (por ejemplo, hiper-, super- alta, tardía ..., pos-). Estos conceptos ayudan a entender cómo esta condición cultural tiene límites porosos y altamente permeables que hacen superfluos los intentos por reificar una categoría que derive en y de una gran narrativa. Las modernidades locales son, en contraste, esos petit rếits que se construyen en el encuentro de procesos local-globales. Hemos tratado de mostrar esto brevemente, tomando ejemplos de un conjunto de trabajos en el que hemos explorado esta nueva posición (Ayora Diaz y Vargas Cetina, editores, en prensa). Finalmente, proponemos que el concepto de modernidad local permite entender los fenómenos derivados de las relaciones de poder establecidas entre sociedades homo/hegemónicas, como les llama Derrida (1997), y sociedades locales.

\section{La modernidad como condición y como experiencia}

La modernidad ha sido caracterizada como un contexto objetivo, como una forma cultural, o como un contexto que contribuye a su vez a la generación de los textos significativos en formas discursivas y en la praxis social. Entre la multiplicidad de conceptos heterogéneos que han sido relacionados con la modernidad (o con la posmodernidad) aquellos que nos permiten caracterizar la experiencia y su forma son: racionalidad, individualización y reflexividad. En lo que sigue procedemos a caracterizar brevemente cada uno de éstos para ilustrar así cómo contribuyen a la comprensión de los fenómenos socioculturales contemporáneos. Sin embargo, en primer lugar, nos parece necesario plantear de manera explícita cómo entendemos la relación entre globalización y modernidad.

¿Es la modernidad una consecuencia de la globalización o, viceversa, la globalización un producto de la modernidad? La respuesta depende, en gran parte, de qué es lo que entendemos por globalización. Aunque algunos autores consideran que la globalización es una consecuencia de la modernidad (Giddens 1990, 1991, Tomlinson 2001), en varios trabajos (por ejemplo Ayora Diaz 1998, 2000, 2003; Vargas Cetina 1999, 2000, 2003) hemos sostenido, de acuerdo con 
Robertson (1992), que la globalización es un proceso de muy larga duración que ha experimentado distintos momentos de aceleración. Si bien Robertson señala que la globalización comienza con la expansión de las grandes religiones, un momento significativo de aceleración —al menos para México y otras regiones de este continente- fue el de la expansión imperial/colonialista de las naciones europeas hacia esta parte del globo. Otros momentos serían, primero, los de las luchas nacionales que surgieron de manera casi simultánea en ambos lados del océano Atlántico; el de la revolución industrial y tecnológica; la Ilustración en el ámbito de la generación de conocimiento y la subordinación de lo estético a lo científico y racional; y, después de la Segunda Guerra Mundial, el desarrollo de nuevas tecnologías y de más veloces formas de comunicación. Cada uno de estos momentos de aceleración ha resultado en la intensificación de lo que Harvey (1991) llama compresión espacio-temporal. Esta conceptualización nos acerca a una comprensión de la globalización y los procesos acompañantes de homoheterogeneización de la cultura como uno solo, de muy larga duración.

La globalización es tanto un complejo proceso multidimensional como un concepto que nos permite entender las interconexiones, las convergencias y las divergencias entre fenómenos de índole social, cultural, económica y política (Robertson 1992, Appadurai 1996). Si bien privilegiamos el análisis de las dinámicas local/ globales que suceden en la esfera sociocultural, reconocemos también la importancia de investigar los entrecruzamientos con procesos y eventos económicos y políticos ya que, en última instancia, éstos también contienen referencias socioculturales (Ayora-Diaz y Vargas Cetina en prensa). En este sentido, la globalización cultural se encuentra ligada a la modernidad (Fornäis 1995). La modernidad, aunque puede ser reducida a las transformaciones tecnológicas y económicas o a las revoluciones políticas con las que se encuentra ligada, es en sí una forma cultural, una compleja red de significados, discursos y prácticas que dan sentido al paso y naturaleza de los cambios experimentados por los sujetos en su vida cotidiana; es ese régimen de significación que permite dar sentido al régimen de producción contemporáneo (Harvey 1991). Los procesos culturales acompañan a, y se corresponden con, los procesos en las otras esferas, sin determinar ni ser determinados los unos por los otros. Aunque la forma cultural que hoy reconocemos como moderna ${ }^{1}$ haya sido gestada, principalmente, en las sociedades europeas occidentales, ella ha sido universalizada y, por ende, localizada de distintas maneras. La modernidad no es ya propiedad de ninguna sociedad nacional.

En otra parte (Ayora Diaz y Vargas Cetina en prensa) hemos mostrado cómo las ideas de modernidad y globalización son entendidas y vividas de forma diferente por grupos locales. En los altos de Cerdeña central, entre las familias pastoriles, la modernidad es percibida como algo deseable que se relaciona con la vida cotidiana. La 'tradición' ha sido vinculada, en la óptica de los sectores gobernantes, a prácticas culturales que inducen a la criminalidad a quienes se niegan a entrar en la modernidad representada por nuevas lógicas y prácticas productivas. El gobierno italiano y el gobierno regional de Cerdeña han tratado de desvincular la producción animal y de derivados de la leche de las prácticas 'tradicionales' representadas por el pastoreo trashumante y el uso de pastos de propiedad comunal, para fomentar la cría de ganado menor y producción lechera sedentarias. Las cooperativas han sido asociadas, en esta región del mundo, con 'la modernidad' porque hacen posible la creación de economías de escala, vinculando las unidades productivas a unidades de procesamiento industrial de carnes y lácteos. La globalización, relacionada en Cerdeña central con la idea de europeización, es vista en estas localidades rurales como algo que permea más bien la esfera del consumo y tiene poca relevancia en términos de la vida cotidiana. 
En gran parte del Chiapas rural, en cambio, la modernización es vista como algo que se relaciona con el acceso a servicios y los hábitos de consumo. En el norte de la selva y en los Altos de Chiapas, por ejemplo, los zapatos deportivos, los aparatos personales de reproducción de música, así como la tecnología relacionada con el video y la red internet son considerados 'modernos', y su uso no se percibe como una forma fundamental de alteración de las maneras locales de vivir. La globalización, en cambio, sobre todo a partir de la caracterización que intelectuales mexicanos y mexicanas y representantes del Ejército Zapatista de Liberación $\mathrm{Na}$ cional (EZLN) han hecho a partir de los noventa, es frecuentemente percibida como algo que se relaciona con políticas económicas internacionales, y con acciones de los gobiernos federal y estatal. La tradición, muchas veces, tiene que ser modificada siguiendo los lineamientos preestablecidos por la sociedad hegemónica. La 'medicina tradicional', que ha sido reducida a la herbolaria y reconocida así como interlocutora por la medicina cosmopolita, es un producto del imaginario moderno que luego ha sido asumido como propio por un número de grupos locales.

Entre los atributos que son reconocidos a la modernidad, en la teoría social en general, merece especial reconocimiento el desarrollo de lo que ha sido llamado 'racionalidad'. Este concepto se refiere a una serie de cambios graduales relacionados con una mayor secularización o desencantamiento de las relaciones sociales y del mundo y con la separación y autonomización de las distintas esferas que componen el mundo de la cotidianidad. Respecto a ambos atributos, las experiencias contemporáneas parecen indicar que ni uno ni otro han sido alcanzados en ningún punto del orbe. Más aún, desde el punto de vista absolutista de los modernizadores, se podría entender que ha ocurrido un retroceso o que la modernidad es, en sí, una fantasía. Latour (1993), por ejemplo, ha llegado a sugerir que nunca hemos sido modernos, porque el trabajo de pu- rificación y diferenciación de las esferas nunca se ha dado y el mundo no ha sido desencantado. El mundo contemporáneo es un mundo de híbridos en el que el trabajo de 'traducción' entre las distintas esferas no ha cesado ni manifiesta una tendencia a ceder.

Aunque autores desde Weber hasta Habermas han dado ya un extensivo tratamiento al tema de la separación de las esferas, nos parece que Lash (1997) presenta un esfuerzo de sistematización útil para entender este fenómeno social. Según Lash, la modernidad se caracteriza por un proceso de diferenciación que implica la separación y autonomía de las distintas esferas: cada ámbito contiene en sí mismo los recursos y las estrategias para resolver racionalmente los problemas que en él se presenten. En este sentido, un problema económico no se resuelve de manera racional, ni rezando, ni con un médico. A cada fin corresponden un número de medios apropiados. Sin embargo, en la posmodernidad, que no sucede a la modernidad sino que se sobrepone y se articula con ella, se da un proceso de des-diferenciación: en sus esfuerzos por encontrar sentido en su vida diaria, los individuos utilizan recursos de distintas esferas para alcanzar un fin que la modernidad localizaría en una sola de ellas. Esta racionalidad, que más recientemente Lash (1999) ha llamado de la tercera modernidad, es una racionalidad alternativa que surge de las condiciones en las que se producen articulaciones local-globales de lo moderno. No es un 'regreso' a las condiciones des-diferenciadas de la premodernidad, sino la creatividad del bricolaje que usa herramientas y recursos de donde sea necesario para alcanzar el objetivo de comprender el mundo que uno habita.

En el libro que hemos dedicado al análisis de la construcción de modernidades locales, Sabina Magliocco (en prensa) describe el proceso que ella llama de 'reclamación del folclore'. Personas estadunidenses de origen italiano, marginadas socialmente a lo largo del siglo veinte - por sus conexiones imaginadas o reales con el mundo rural y campesino (con prácticas mágicas y crimina- 
les originadas, según ese imaginario, en la cultura campesina de Italia) — buscan reconstruir sus identidades mediante la resignificación de la magia dentro de la creciente constelación de religiones 'New Age'. Según Magliocco, gracias al modernismo multicultural y la relativización de las cosmovisiones, los sujetos pueden redefinirse de manera positiva. En ese mismo volumen, Igor Ayora Diaz (en prensa) sostiene que en Chiapas las medicinas herbolarias son formas 'modernas' de medicina 'tradicional' que resultan de las estructuras desiguales de poder en las que se encuentran inscritas las medicinas locales y la medicina cosmopolita. En esas estructuras la última tiene la capacidad de definir y legitimar como aceptable el conocimiento herbolario (y por ende fomentarlo) y silenciar las prácticas que se alejan de la visión cientificista de la medicina.

Robertson (1992) ha llamado 'de incertidumbre' el momento contemporáneo de globalización cultural. Este es un tiempo en el que las certezas han comenzado a derrumbarse, en parte por la relativización de las formas, valores y cosmovisiones culturales que resultan de la experiencia del mundo global, y en parte de lo que se ha llamado reflexividad social. Lash $(1997,1999)$, por ejemplo, afirma que no son solamente los individuos quienes pueden 'reflexionar' sobre sus propias experiencias y condiciones de vida, sino que también las instituciones sociales contienen en sílos mecanismos que les permiten organizar el conocimiento acerca de su propia generación y reproducción. Las instituciones y las prácticas sociales son, en este sentido, desnaturalizadas; su carácter y sus orígenes sociales y culturales se revelan y transparentan. En condiciones de incertidumbre y de reflexividad, los sujetos sociales pierden los referentes que pudieron haber hecho obligatoria la adherencia a una cosmovisión absolutista o totalizante: el progreso no puede tener las mismas características para todas las sociedades; la modemidad puede ser vivida de muchas más que una sola manera. Se generan así las posibilidades para una nueva forma de individualismo en la que el sujeto se encuentra ante múltiples formas alternativas de vida. El estilo de vida se convierte entonces en una forma individual de manifestar los valores y preferencias sin que necesariamente el sujeto se vea obligado a mantener una coherencia o sistematicidad comprehensiva (Giddens 1991, Featherstone 1991). Este es el individualismo que permite la formación en la sociedad contemporánea de comunidades, llamadas por varios autores, 'de sentido', 'de estilo de vida', 'de consumo'; comunidades frágiles, efímeras, que permiten construir sentidos particulares y rechazan la universalización de criterios absolutos acerca de la dirección del cambio social o del 'progreso' mismo.

Desde este punto de vista, preguntas como si es posible o no universalizar las actuales formas de vida de las comunidades indígenas, inclusive cuando se piensa en extenderlas a otros grupos también considerados indígenas, carecen de sentido: las personas en las comunidades indígenas y cada uno de los individuos que viven en lo que actualmente es la república mexicana tienen ante sí múltiples posibilidades de estilos de vida, formas de ver el mundo y de verse a sí mismos y sí mismas en la sociedad y el mundo (las posibles formasde-estar-en-el-mundo se fragmentan y multiplican). Cada cual está tomando los elementos que puede y quiere adoptar, incluso de entre los que percibe como 'modernos', 'antiguos', 'tradicionales' o 'posmodernos', para construir su propia trayectoria de vida. Aunque las y los teóricos de la posmodernidad descubrieron recientemente esta constante mezcla de elementos de lo que aparecía como diversas temporalidades ('tradición' y 'modernidad'), la etnografía ha dado cuenta sistemáticamente de la constante hibridación entre lo conocido y lo que se va conociendo. En este sentido aquellos análisis que proponen que la hibridación es la adopción de elementos modernos por sociedades tradicionales yerran desde su mismo punto de partida, pues la vida cotidiana nunca ha sido estática en ninguna sociedad, y la tradición misma es incomprensible fuera de su con- 
ceptualización automática como parte de la modernidad. Tomando como punto de partida esta fragmentación cultural, social, política y económica, del mundo global y de múltiples maneras de ser modernos, Gabriela Vargas Cetina (en prensa) argumenta que los sistemas legales consuetudinarios y 'tradicionales' de la región Selva de Chiapas forman parte integral de la lógica jurídica estatal y federal. Ésta tiene raíces en la Colonia, pero se ha desarrollado y generalizado mediante la legislación republicana, incluyendo la Constitución, documento fundador del México moderno, y demás leyes de vigencia contemporánea.

Encontramos necesario trascender el carácter absoluto (y absolutista) y espacio-temporal del concepto de modernidad. No podemos ya proponer y aceptar para nuestros análisis una modernización lineal y homogeneizante global, sin hacernos cómplices de los procesos de neocolonialismo cultural que esta visión de la modernidad implica. En vez de una sola modernidad, como anotamos más arriba, necesitamos pensar en una multiplicidad de modernidades locales cuyas formas son diversas y heterogéneas y que resultan de combinaciones únicas entre elementos culturales universalizantes y aquellos particulares, locales. Más aún, necesitamos partir de la idea misma de modernidad como un campo político en el que constantemente se libran batallas por el control de la opinión pública y por la legitimación de núcleos de ideas, muchas veces antagónicos, sobre el rumbo de regiones y naciones. Blair Rutherford (en prensa), por ejemplo, examina las ambivalencias sobre la modernidad originadas con la independencia de Zimbabwe; en particular, analiza el hecho de que las y los colonizadores blancos, quienes antes de la independencia eran el ejemplo de lo moderno, han pasado a ser vistos como anacrónicos y son ahora las y los miembros de la elite local (africana) quienes buscan resignificarse como modernos integrantes de la economía global, presentando a las élites blancas como el símbolo de la tradición que hay que dejar atrás.
En los múltiples procesos de apropiación de la modernidad, sus instituciones se transforman en maneras no anticipadas por las y los teóricos y apologistas de la modernidad.

\section{Modernidades locales: la fragmentación del mundo}

\author{
En todas partes, en cada sitio nacional/ cultural, \\ la modernidad no es una sino muchas; \\ la modernidad no es nueva, sino vieja y familiar; \\ la modernidad es incompleta y necesariamente es así.
}

(Gaonkar 1999:18)

La idea de que la modernización (igual que el 'progreso') es un proceso único y monolítico de transformación lineal ha sido cuestionada ya desde hace más de una década (véase Appadurai 1991, para una aproximación temprana a la noción de modernidades alternativas). Sin embargo, sólo recientemente han comenzado esfuerzos por sistematizar el contenido del concepto de modernidad alternativa. Lash (1999), por ejemplo, distingue entre tres modernidades: la primera, ligada a la Ilustración, es la modernidad racional, tecnológica, del progreso, un proceso que excluye al Otro; es la modernidad de la mismidad (sameness). La segunda modernidad, gestada en el siglo veinte, es la modernidad de la fragmentación social, de los movimientos de lucha identitarios, incluyendo distintos feminismos, luchas étnicas, nacionalistas, movimientos gay o del tercer sexo, y movimientos religiosos fundamentalistas. Es la modernidad de la otredad (otherness). ${ }^{2}$ Entre estas modernidades, Lash identifica la otra modernidad, la racionalidad diferente, aquella que corresponde a la praxis social al interior de cada grupo. Es la modernidad de la diferencia, aquella en la que los sujetos establecen un puente entre las demandas de la primera y de la segunda modernidad. En este sentido, en la alta modernidad, supermodernidad, hipermodernidad, modernidad tardía, posmodernidad, o como se le prefiera llamar, el movimiento global 
homogeneizador (la modernidad de la mismidad), lleva en sí mismo las semillas de la diversidad (desigualdades de poder, colonialismo cultural, sistemas de ideas sobre las diferencias de género, sexuales, étnicas, religiosas, los derechos humanos - la modernidad de la otredad). Sin embargo, es en la praxis local donde los sujetos se apropian de elementos, tanto de la primera como de la segunda modernidad, creando así las condiciones para la gestación de la diferencia. En pocas palabras, la tendencia homogeneizadora de la modernidad globalizada resulta en la heterogeneización de las sociedades locales, fragmentando el mundo en múltiples formas de modernidad local.

En un número especial dedicado al tema, Gaonkar (1999) reconoce que hablar de modernidades alternativas es, en cierta forma, reconocer lo ineluctable de la modernidad; de una modernidad nacida en el occidente europeo, pero ya establecida en el resto del mundo. Sugiere que es importante reconocer dos aspectos de la modernidad: la modernización societal y la modernidad cultural. La primera está caracterizada por el desarrollo inexorable, en el lado cognitivo, de los sistemas científicos de conocimiento, la secularización de la sociedad, la separación entre valor y hecho, el individualismo y la hegemonía de la doctrina del progreso. En su lado social, por la aparición e institucionalización de economías de mercado, por la administración burocrática de los estados nación, por mayor movilidad social, por el afianzamiento del orden legal, el alfabetismo y la urbanización (Gaonkar 1999:2). La segunda, la modernidad cultural, es una respuesta de las elites culturales a la modernidad societal. Ante las transformaciones dramáticas en el orden social, el modernismo, como respuesta estética, política y moral se basaba en un cuidado del yo: el individuo auto-cultivado rechazaría la lógica racional, monetaria, y privilegiaría la dimensión estética de la vida cotidiana. Según Gaonkar, esta sería una respuesta organizada de las élites en los distintos movimientos de avant-garde que posteriormente se extenderían al resto de la sociedad (por ejemplo a través, entre otras cosas, de los medios de comunicación).

Fue el grupo de trabajos editado por Gaonkar, incluyendo su propio ensayo, el que impulsó a nuestro grupo de trabajo a buscar formas de conceptuar, de manera más etnográficamente sensible, la relación entre los imaginarios locales sobre la modernidad y las relaciones de poder tanto locales como nacionales y transnacionales en las que estos imaginarios cobran sentido, son apropiados y utilizados en la vida cotidiana local. Desde nuestro punto de vista, la propuesta de Gaonkar (1999) es útil en tanto que nos ayuda a matizar aún más el sentido de modernidad y apunta en la dirección del concepto de modernidades locales. Sin embargo, Gaonkar le concede un papel privilegiado a las élites culturales en el proceso de generación de las modernidades alternativas.

Taylor (1999), por su parte, distingue entre dos formas de entender la modernidad: una, hegemónica hasta el momento, es la comprensión acultural de la modernidad. Según esta comprensión, la modernidad es inexorable y se define como un proceso culturalmente neutro. Modernidad sería entonces:

El crecimiento de la conciencia científica, el desarrollo de una perspectiva secular, el crecimiento de la racionalidad instrumental y una distinción cada vez más clara entre la búsqueda de los hechos y la evaluación. ... Las transformaciones, incluyendo las intelectuales, son vistas como resultando de una mayor movilidad, la concentración de la población, la industrialización y demás. En todos estos casos, la modernidad se concibe como un paquete de transformaciones que toda y cada cultura debe atravesar - y que todas ellas probablemente estarán forzadas a vivir. (Taylor 1999:154)

Un problema con la teoría acultural de la modernidad, sugiere Taylor, es que asume que la cultura moder- 
na del Atlántico norte es la cultura. Al condensar todos los valores y formas universales, se convierte en la meta a la que todas las demás sociedades deben llegar. Sin embargo, en la sociedad contemporánea, como ya se ha mencionado, las formas sociales y culturales se han relativizado ante el surgimiento de la conciencia global. Hoy reconocemos que la cultura del Atlántico norte es una más entre tantas culturas. En este sentido Taylor sugiere que la modernidad, como forma cultural, al adoptarse en otras sociedades, derivará en algo distinto como resultado de puntos de partida distintos: "La comprensión de distintas culturas de lo que es la persona, de las relaciones sociales, los estados mentales, lo bueno y lo malo, las virtudes y los vicios, lo sagrado y lo profano, es seguramente distinta. El futuro del mundo será que todas las sociedades cambiarán, en sus instituciones y perspectiva, y algunos de estos cambios serán paralelos pero no convergerán, ya que nuevas diferencias surgirán de las viejas" (1999:162). Para Taylor (1999), por tanto, las modernidades alternativas son formas heterogéneas de la modernidad cultural. Una vez más, encontramos un límite en esta comprensión de modernidades alternativas: se entiende como modernidad alternativa el resultado de un proceso de modemización que es a su vez consecuencia de la implementación en distintos lugares de un grupo; prácticas, valores y cosmovisión cuya meta final es modificada por el punto de partida cultural. Las modernidades alternativas serían así formas culturalmente distorsionadas de la modernidad del Atlántico norte. La perspectiva de Taylor, como las otras hasta aquí mencionadas, vuelve a reificar temporalmente el concepto de modernidad. Sin embargo, las aportaciones al debate por parte de Lash, Gaonkar y Taylor nos permiten comenzar a plantearnos el concepto de modernidades locales.

El concepto de modernidad local nos debe apartar de pensar en la modernidad como una serie de atributos sociales, culturales —estéticos, morales y políticosestablecidos que (con mayor o menor transparencia) son derivados del grupo de culturas del Atlántico norte. Aceptar estos criterios definitorios, aun cuando sea como herramienta analítica, es, en gran medida, contribuir a las nuevas formas de colonialismo cultural en las que la academia se encuentra frecuentemente involucrada. Nos parece necesario tener siempre presente que la sociedad moderna no implica, requiere, ni necesita, la desaparición de otras formas culturales, económicas o políticas. Por el contrario, encuentra su sentido en la existencia de esas otras formas y la referencia que hace a ellas para poder crear su propia identidad. Los grupos sociales auto- y hetero- denominados 'tradicional', 'moderno' y 'posmoderno' son en gran medida el resultado de imaginarios socioculturales complejos que entran en un juego de efectos especulares. Las luces y sombras que cada uno refleja contribuyen a definir su propia imagen e identidad. Así, cada concepto articula una serie de presencias y ausencias: visión secular o visión religiosa, cambio acelerado o estasis social, rural o urbano, Gemeinschafto Gesellschaft, intercambio de dones o mercado, cooperación o asociación, encadenado a la sociedad o individualista, creencias supersticiosas o conocimientos científicos, y otras más. Dentro de este juego, la ubicación espacio-temporal de un grupo en la tradición o en la modernidad o posmodernidad, implica la legitimación de una estructura de poder y subordinación en la que los grupos modernos controlan los recursos del Estado y de la identidad nacional.

Con la globalización cultural necesitamos reconocer la coexistencia y simultaneidad de tendencias hacia la universalización de lo particulary la particularización de lo universal. No es posible dejar de reconocer el impulso colonialista cultural que la forma moderna tiene en escala global. En este sentido, la modernidad es una forma homo/ hegemónica que élites políticas y económicas buscan expandir de forma global. Sin embargo, localmente existen procesos de rechazo, imposición, adopción, adaptación y re-significación de formas homo/ hegemónicas. En un mundo de modernidad 
globalizada, cada sociedad local se esfuerza, en mayor o menor medida, por re-crearse y re-definirse utilizando de manera creativa elementos tanto locales como globales. Por supuesto, esta re-creación, re-definición y re-significación se da en un contexto de relaciones de poder económico, político y militar que crea las condiciones para que, según el lugar y la sociedad, los grupos locales puedan construirse a sí mismos como formas socioculturales distintivas. Este es un proceso variable según el grupo del que se trate y el resultado es, en consecuencia, diverso también. Las comunidades locales y la 'sociedad global' no operan ni pueden haber operado de manera autónoma para constituirse a sí mismas.

Si aceptamos estas premisas, negamos entonces los atributos temporales y espaciales que han caracterizado a la mayor parte de los debates sobre cuál es la temporalidad que encierra y corresponde a la ‘tradición', la 'modernidad' y la 'posmodernidad' para apuntar hacia el contenido político del concepto de modernidad cuando es usado desde el Estado y las instancias de intervención social y cultural. Asimismo, si usamos el concepto de modernidad en el sentido dado a éste por las personas que en distintos puntos del mundo eligen entre prácticas 'más modernas' y 'más tradicionales' para cocinar, atender sus aflicciones físicas y emocionales, vestirse y adornarse, construir y amueblar sus viviendas, desplazarse, o llevar a cabo cualquier actividad cotidiana (incluyendo la expresión individual u organizada de sus descontentos), entonces apuntamos a la percepción de modernidad como sólo una de las maneras posibles en las que las personas pueden categorizar los objetos y procesos de su experiencia diaria.

Desde esta perspectiva, tiene poco sentido preguntarse en forma abstracta, por ejemplo, si el movimiento zapatista es una forma de protesta tradicional, moderna o postmoderna, y pasa a ser más importante conocer cómo conceptualizan el movimiento las y los múltiples actores individuales y colectivos que han tomado parte en él (ver Harvey 1998; Leyva 1999; Nash 2001). Es claro, desde nuestro punto de vista tal como aquí lo hemos esbozado, que las acciones del estado, la 'modernización' del campo y, en este caso, de las zonas en las que la lucha zapatista se ha manifestado, intentan tener un efecto de despolitización, en la forma ya señalada por Ferguson (1994) y otros. Desde la experiencia vivida de la modernidad, tal como se da en Chiapas, la modernización puede implicar cosas muy diferentes. Éstas pueden ir desde la adopción de internet como un vehículo de comunicación con otros grupos y con el resto del mundo, al uso de cámaras de fotografía y video por la gente local — que finalmente son elementos aislados que no transforman radicalmente, por sí mismos, la vida cotidiana local.

Las sociedades se encuentran todas como partícipes desiguales en un mundo global; pero todas ellas son cotemporales y comparten el mismo espacio terráqueo (aunque, por supuesto, sus ligares sean distintos). Existen sociedades en las que la forma de producción es campesina, que demográficamente son rurales, cuya población es mayoritariamente indígena, cuyo acceso a tecnología de punta es limitado, con acceso también limitado al mercado mundial, en las que predominan estructuras locales de poder político y religioso que la sociedad homo/ hegemónica no reconoce como democrático, donde, de acuerdo con esa sociedad homo/ hegemónica, no se respetan adecuadamente los derechos humanos, sociedades que han sido marginadas en el reparto de beneficios del estado de bienestar (hoy liquidado por las políticas neoliberales). Estas son también sociedades modernas. El hecho de que hayan sido histórica y estructuralmente subordinadas a otras formas socioculturales no niega su coevalidad. En estas sociedades, en mayor o menor medida, los valores, las prácticas, las tecnologías, elementos de la cosmovisión que hoy definimos como modernos se encuentran presentes, aun cuando estén y sean continuamente re-significados. Por otra parte, aspectos locales que son corrientemente identificados como tradicionales tienen hoy 
un sentido moderno; aunque esta modernidad sea distinta de la modernidad tradicionalmente entendida. En el mundo postcolonial, es importante pensar que las modernidades son múltiples y locales y no tienen por qué compartir una serie de atributos fijos y predeterminados por la cultura homo/ hegemónica.

\section{Conclusión: El más acá de la modernidad}

Con estas reflexiones queremos apuntar hacia dos conclusiones importantes: la primera es que en la antropología los conceptos de tradición, modernidad, posmodernidad, y conceptos relacionados, tienen en este momento poca utilidad en su forma más abstracta. Puesto que son usados con implicaciones retóricas de un desplazamiento temporal tienen la impronta de la visión colonial y colonizadora, y en general llevan consigo la perspectiva 'de arriba hacia abajo' y las pretensiones de control del aparato de Estado.

La segunda es que ahora es necesario convertir nuestras certezas en preguntas y pasar de asumir ideas generales y abstractas sobre la modernidad a las formas en las que sociedades, grupos e individuos entienden y viven la tradición, modernidad o posmodernidad en las diferentes partes de nuestro mundo, que sigue siendo uno sólo y que se desplaza en el tiempo en forma simultánea, de un pasado a un presente y a un futuro común. Es a esta transformación de la modernidad y la postmodernidad en preguntas de investigación que se refiere el concepto de 'modernidades locales' que estamos proponiendo.

No consideramos que sea posible desechar totalmente las ideas de tradición, modernidad o posmodernidad porque no podemos negar la forma en la que la gente en todas partes entiende y en-corpora estos conceptos. Queremos, sin embargo, señalar un camino por el que podemos comenzar a salir del actual bache conceptual: México, como todas las naciones y culturas del mundo, es una sociedad nacional moderna en tanto que quienes vivimos en su amplio territorio habitamos de maneras diferentes en el más acá de nuestra propia modernidad.

\section{Agradecimientos}

Agradecemos a Witold Jacorzynski el habernos invitado a debatir en forma escrita las ideas de modernidad, posmodernidad y localidad y su importancia en la antropología contemporánea. Gracias a Blair Rutherford, Sabina Magliocco, y Patricia Fortuny, integrantes de nuestro grupo de trabajo sobre etnografía de la modernidad, por discutir con nosotros estos temas a lo largo de varios años. Gracias también por la discusión y las sugerencias a los participantes en el simposio sobre Globalization and its Discontents (Allan Burns, Dipesh Chakrabarty, Eric Fassin y Reiner Kraetsch) organizado por la Facultad de Humanidades de la Universidad de Florida en 2001, en donde presentamos por primera vez nuestras ideas conceptuales sobre modernidades locales. Gracias, finalmente, a José Luis Escalona y a Axel Köhler por haber comentado una primera versión de este escrito. Cualquier omisión, falta de claridad o error de interpretación, sin embargo, es solamente atribuible a nosotros dos, autor y autora de este artículo.

\section{Notas}

${ }^{1}$ Distintos autores (Fornäis 1995, Giddens 1991, Habermas 1997) han reconocido que 'modernidad' es un término utilizado por distintos grupos para distinguirse de sus Otros a quienes se les posiciona en el pasado de la 'tradición'. Habermas (1997) identifica un uso temprano en el siglo v de nuestra era para distinguir a la sociedad cristiana de la pagana.

${ }^{2}$ Tal como en el caso de la premodernidad, la modernidad y la posmodernidad, que deben ser entendidas como sobrepuestas, la primera, segunda y tercera modernidad no estarían ligadas a temporalidades concretas y precisas. Uno puede encontrar sus raíces en movimientos filosóficos e intelectuales de distintas épocas. En este caso también, la discusión sobre si cada una 
de estas modernidades comienza en tal o cual año es otro callejón sin salida conceptual.

\section{Bibliografía}

Appadurai, Arjun, 1991, "Global Ethnoscapes: Notes and Queries for a Transnational Anthropology”. En Richard G. Fox (ed.) Recapturing Anthropology. Working in the Present, School of American Research, Santa Fe.

—, 1996, Modernity at Large. Cultural Dimensions of Globalization, University of Minnesota Press, Minneapolis.

Ayora Diaz, Steffan Igor, 1998, "Globalization, Rationality and Medicine: Local Medicines' Struggle for Recognition in Highland Chiapas", en Urban Anthropology and Studies of Cultural Systems and World Economic Development, 27(2):165-195. —, 2000, "Globalización cultural y medicina. Medicinas locales y medicina cosmopolita en Chiapas", en Carmen Bueno Castellanos (Coord.) Globalización: Una cuestión antropológica, CIESAS/ Miguel Ángel Porrúa.

—, 2002, Globalización, conocimiento y poder. Medicinas locales y sus luchas por el reconocimiento en Chiapas, Plaza y Valdés/ Universidad Autónoma de Yucatán, México, DF y Mérida.

—, 2003, "Re/creaciones de la comunidad: Espacios translocales en la globalización". Cuadernos de Bioética Año 10, Núm. 7:27-45.

-, en prensa, "La modernidad del conocimiento local: La medicina herbolaria y el escrito oculto de las medicinas locales en Chiapas" en Steffan Igor Ayora Diaz y Gabriela Vargas Cetina (Coords). Modernidades Locales. Etnografía del presente múltiple, Universidad Autónoma de Yucatán, Mérida.

—, y Gabriela Vargas Cetina, en prensa, "Globalización y localización: La producción de las modernidades locales" en Steffan Igor Ayora Diaz y Gabriela Vargas Cetina (editores) Modernidades locales. Etnografía del presente múltiple, Universidad Autónoma de Yucatán, Mérida.

—, y Gabriela Vargas Cetina (editores), en prensa, Modernidades locales. Etnografía del presente múltiple. Universidad Autónoma de Yucatán, Mérida.

Comaroff, Jean y John Comaroff, 1993, "Introduction" en Jean Comaroff y John Comaroff (editores) Modernity and its Malcontents. Ritual and Power in Postcolonial Africa, University of Chicago Press, Chicago.

Derrida, Jacques, 1997/1996, El monolingüismo del otro o la prótesis de origen, Manantial, SRL, Buenos Aires.
Englund, Harry y James Leach, 2000, "Ethnography and the Meta-Narratives of Modernity", Current Anthropology, 41(2):225-248.

Escobar, Arturo, 1994, Encountering Development. The Making and Unmaking of the Third World, Princeton University Press, Nueva Jersey.

Fabian, Johannes, 1983, Time and the Other. How Anthropology Makes Its Other, Indiana University Press, Bloomington.

Fairhead, James, 2000, "Development Discourse and its Subversion: Decivilisation, Depoliticisation and Dispossession in West Africa”, en Alberto Arce y Norman Long, (eds.) Anthropology, Development and Modernities, Routledge, Londres y Nueva York.

Featherstone, Mike, 1991, Consumer Culture and Postmodernism, Sage, Londres.

Ferguson, James, 1994, The Anti-Politics Machine. "Development", Depolitization and Bureaucratic Power in Lesotho, University of Minnesota Press, Minneapolis.

Fornäis, Johan, 1995, Cultural Theory and Late Modernity, Sage, Londres.

Gaonkar, Dilip Parameshwar, 1999, “On Alternative Modernities", en Public Culture, 11(1):1-18.

Giddens, Anthony, 1990, The Consequences of Modernity, Stanford University Press, Stanford.

-, 1991, Modernity and Self Identity. Self and Society in Late Modern Age, Stanford University Press, Stanford.

Habermas, Jürgen, 1997/1981, "Modernity: An Unfinished Project”, en Maurizio P. d'Entrèves y Seyla Benhabib (editores) Habermas and the Unfinished Project of Modernity. Critical Essays on The Philosophical Discourse of Modernity, The MIT Press, Cambridge.

Harvey, David, 1991, The Condition of Postmodernity. An Enquiry into the Origins of Culture Change, Blackwell, Oxford. Harvey, Neil, 1998, The Chiapas Rebellion. The Struggle for Land and Democracy, Duke University Press, Durham.

Hobart, Mark E. (editor), 1993, An Anthropological Critique of Development, Routledge, Londres y Nueva York.

Knauft, Bruce M. (editor), 2002, Critically Modern. Alternatives, Alterities, Anthropologies, Indiana University Press, Bloomington.

Lash, Scott, 1997/1990, Sociología del posmodernismo, Amorrortu, Buenos Aires.

—, 1999, Another Modernity. A Different Rationality, Blackwell, Oxford. 
Latham, Michael E., 2000, Modernization as an Ideology. American Social Science and "Nation Building" in the Kennedy Era, The University of North Carolina Press, Chapel Hill.

Latour, Bruno, 1993, We Have Never Been Modern, Harvard University Press, Cambridge.

Leys, Colin, 1996/1977, The Rise and Fall of Development Theory, Indiana University Press, Bloomington.

Leyva, Xóchitl, 1999, "De las cañadas a Europa: Niveles, actores y discursos del Nuevo Movimiento Zapatista (NMZ) (19941997)", en Desacatos 1:56-76.

Magliocco, Sabina, en prensa, "Imaginando la strega: La reclamación del folclore y la construcción de la brujería ítaloestadounidense" Steffan Igor Ayora Diaz y Gabriela Vargas Cetina (editores) Modernidades locales. Etnografía del presente múltiple, Universidad Autónoma de Yucatán, Mérida.

Marcus, George E., 1998, Ethnography Through Thick and Thin, Princeton University Press, Princeton.

Moreiras, Alberto, 1998, "Global Fragments: A Second Latinamericanism”, en Frederic Jameson y Masao Miyoshi (editores) The Cultures of Globalization, Duke University Press, Durham.

Nash, June, 2001, Maya Visions: The Quest for Autonomy in an Age of Globalization, Routledge, Londres y Nueva York.

Robertson, Roland, 1992, Globalization. Social Theory and Global Culture, Sage, Londres.

Rushdie, Salman, 2001, Fury. A Novel, Random House, Nueva York.

Rutherford, Blair, en prensa, "Públicos blancos, nación negra: Granjeros, trabajadores agrícolas y sociedad civil en Zimbabwe" en Steffan Igor Ayora Diaz y Gabriela Vargas Cetina (editores) Modernidades locales. Etnografía del presente múltiple. Universidad Autónoma de Yucatán, Mérida.

Said Edward W., 1978, Orientalism, Random House, Nueva York.

Taylor, Charles, 1999, "Two Theories of Modernity", Public Culture, 11(1):153-174.

Tomlinson, John, 2001/1999, Globalización y Cultura, Oxford University Press, México, DF.

Vargas Cetina, Gabriela, 1999, “Flexible Looms: Weavers' Organizations in Chiapas, Mexico", en Urban Anthropology and Studies of Cultural Systems and World Economic Development 28(3 and 4): 299-326.

—, 2000, "El Movimiento Cooperativo Internacional Localizado", en Carmen Bueno Castellanos (coordinadora) Globalización, Una Cuestión Antropológica, CIESAS/Universidad Iberoamericana, México, DF.

—, 2003, "La asociación efímera. Repensando el concepto de comunidad desde la literatura cyberpunk". Cuadernos de Bioética Núm. 11. Publicado en Internet en http:/ / www.bioetica.org/ doctrina 38.htm.

—, en prensa, "Tradiciones modernas: Solución local de conflictos en el norte de la Selva de Chiapas" en Steffan Igor Ayora Diaz y Gabriela Vargas Cetina (editores) Modernidades Locales. Etnografía del Presente Múltiple, Universidad Autónoma de Yucatán, Mérida. 